\title{
Explicit expanders of every degree and size
}

\author{
Noga Alon *
}

\begin{abstract}
An $(n, d, \lambda)$-graph is a $d$ regular graph on $n$ vertices in which the absolute value of any nontrivial eigenvalue is at most $\lambda$. For any constant $d \geq 3, \epsilon>0$ and all sufficiently large $n$ we show that there is a deterministic poly(n) time algorithm that outputs an ( $n, d, \lambda$ )-graph (on exactly $n$ vertices) with $\lambda \leq 2 \sqrt{d-1}+\epsilon$. For any $d=p+2$ with $p \equiv$ $1 \bmod 4$ prime and all sufficiently large $n$, we describe a strongly explicit construction of an $(n, d, \lambda)$-graph (on exactly $n$ vertices) with $\lambda \leq \sqrt{2(d-1)}+\sqrt{d-2}+o(1)(<$ $(1+\sqrt{2}) \sqrt{d-1}+o(1))$, with the $o(1)$ term tending to 0 as $n$ tends to infinity. For every $\epsilon>0, d>d_{0}(\epsilon)$ and $n>n_{0}(d, \epsilon)$ we present a strongly explicit construction of an $(m, d, \lambda)$-graph with $\lambda<(2+\epsilon) \sqrt{d}$ and $m=n+o(n)$. All constructions are obtained by starting with known ones of Ramanujan or nearly Ramanujan graphs, modifying or packing them in an appropriate way. The spectral analysis relies on the delocalization of eigenvectors of regular graphs in cycle-free neighborhoods.
\end{abstract}

\section{Introduction}

An $(n, d, \lambda)$-graph is a $d$-regular graph on $n$ vertices in which the absolute value of every nontrivial eigenvalue is at most $\lambda$. This notation was introduced by the author in the early 90 s motivated by the fact that such graphs in which $\lambda$ is much smaller than $d$ exhibit strong expansion and quasi-random properties, see [1], 2], 14].

It is well known (see [1, [22, 9]) that if an $(n, d, \lambda)$-graph exists then $\lambda \geq 2 \sqrt{d-1}-$ $O\left(1 / \log ^{2} n\right)$. An $(n, d, \lambda)$-graph is called (two-sided) Ramanujan if $\lambda \leq 2 \sqrt{d-1}$.

Lubotzky, Phillips and Sarnak [16, and independently Margulis [17] proved that for every prime $p$ which is 1 modulo 4 there are infinite families of $d$-regular Ramanujan graphs. Friedman [10] (see also [6] for a simpler proof) proved the existence of near

*Department of Mathematics, Princeton University, Princeton, NJ 08544, USA and Schools of Mathematics and Computer Science, Tel Aviv University, Tel Aviv 69978, Israel. Email: nogaa@tau.ac.il. Research supported in part by NSF grant DMS-1855464, ISF grant 281/17, BSF grant 2018267 and the Simons Foundation. 
Ramanujan graphs of every degree and every (large) admissible size. Indeed, establishing a conjecture of the present author he proved that a random $d$-regular graph on $n$ vertices is, with high probability, an $(n, d, \lambda)$-graph for $\lambda=2 \sqrt{d-1}+o(1)$, where the $o(1)$-term tends to zero as $n$ tends to infinity.

For applications, however, (see, e.g., [12] and its references for many of those) it is desirable to have explicit constructions of such graphs. It is also sometime desirable to have explicit constructions with specified degrees and number of vertices, (see, for example, 21] for a recent example). A construction is called explicit if there is a deterministic polynomial time algorithm that, given $n$ and $d$, produces an $(n, d, \lambda)$-graph (or an $(n(1+o(1)), d, \lambda)$ graph). It is strongly explicit if the adjacency list of any given vertex can be produced in time polylog$(n)$. The construction of [16], and that of [17] are strongly explicit 1, providing Cayley graphs of $S L\left(2, F_{q}\right)$, but work only for degrees that are $p+1$ for primes $p \equiv 1 \bmod 4$ and for numbers of vertices that are of the form $q\left(q^{2}-1\right) / 2$ for primes $q$ which are 1 modulo 4 so that $p$ is a quadratic residue modulo $p$. Morgenstern [19] gave a strongly explicit construction for every degree which is a prime power plus 1 , but the possible numbers of vertices obtained are sparser. An observation in [7] provides strongly explicit families of $(n, d, \lambda)$-graphs with $\lambda \leq O\left(d^{0.525}\right)$ for infinitely many values of $n$ (but not for every $n$ ). Similarly, the method in [23] and its improvement in [5] provide strongly explicit families with $\lambda \leq O\left(d^{1 / 2+o(1)}\right)$ (for infinitely many, but not for all $n$ ). The results of [20] together with those of [8] and an observation of Srivastava (cf. [18]) give explicit, but not strongly explicit $(n, d, \lambda)$-graphs for all admissible $d$ and $n$ with $\lambda \leq 4 \sqrt{d-1}$. In a recent work of Mohanty, O'Donnell and Paredes [18] the authors describe an explicit (but not strongly explicit) construction of $(n, d, \lambda)$-graphs for every $d$, where $\lambda=2 \sqrt{d-1}+o(1)$ and the $o(1)$-term tends to 0 as $n$ tends to infinity. This, again, works for infinitely many values of $n$, but not for all $n$.

In the present short paper we describe improved explicit and strongly explicit constructions of near Ramanujan graphs of all degrees and (large) number of vertices. The first result is a (slightly improved version of an) observation I mentioned in several lectures in the 90s that, as far as I know, has never appeared in print. Although it is very simple, the parameters it provides are far better than the ones obtained from the constructions in [7, [23], [5], and I therefore decided to include it here.

Proposition 1.1. For every degree $d$ there is a strongly explicit constructions of $(n, d, \lambda)$ graphs where $\lambda \leq\left(2+o_{d}(1)\right) \sqrt{d}$, the $o_{d}(1)$-term tends to zero as d tends to infinity, and

\footnotetext{
${ }^{1}$ Though they require to find a large prime in a prescribed range. This can be done efficiently using randomization, but can also be avoided. More details appear in Section 2
} 
the possible values of $n$ form a sequence in which the ratio between consecutive terms tends to 1 .

Note that this means that for every desired number of vertices $n$ and any desired degree $d$, there is a strongly explicit construction of an $\left(n\left(1+o_{n}(1)\right), d, \lambda\right)$-graph with $\lambda \leq\left(2+o_{d}(1)\right) \sqrt{d}$. Here the term $o_{n}(1)$ tends to zero as $n$ tends to infinity and the $o_{d}(1)$-term tends to zero as $d$ tends to infinity.

The next result provides strongly explicit constructions of $(n, d, \lambda)$ graphs for degrees $d=p+2$ with $p$ being a prime congruent to 1 modulo 4 , for any desired (large) number of vertices.

Theorem 1.2. For any prime $p \equiv 1 \bmod 4$ and every sufficiently large $n$ there is a strongly explicit construction of an $(n, d, \lambda)$-graph (on exactly $n$ vertices), where $d=p+2$ and $\lambda \leq \sqrt{2(d-1)}+\sqrt{d-1}+o(1)<(1+\sqrt{2}) \sqrt{d-1}+o(1)$, and the $o(1)$-term tends to zero as $n$ tends to infinity.

It is worth noting that here we allow to have at most one loop in every vertex, with the convention that a loop adds one to the degree (otherwise we must have an even number of vertices as the degree of regularity is odd). For even $n$ we can replace the loops by a matching with no loss in the spectral estimate.

If an explicit, rather than strongly explicit construction suffices, we can combine a variant of our method with the new result of [18 to get the following.

Theorem 1.3. For every degree $d$, every $\epsilon$ and all sufficiently large $n \geq n_{0}(d, \epsilon)$, where $n d$ is even, there is an explicit construction of an $(n, d, \lambda)$-graph with $\lambda \leq 2 \sqrt{d-1}+\epsilon$.

The construction in the proof of Proposition 1.1 is a simple packing of known Ramanujan graphs on the same set of vertices. A crucial point is that these constructions are all Cayley graphs of the same group, so one can simply take a union of the corresponding generating sets. The proofs of Theorem 1.2 and 1.3 require more work. Here too the idea is to start from a known Ramanujan or nearly Ramanujan graph and modify it in an appropriate way. In the proof of Theorem 1.2 we add vertices connected to arbitrary disjoint sets of neighbors, adding loops (or a matching) to keep the graph regular. The eigenvalues are then estimated by their variational definition. In the construction for Theorem 1.3 we omit carefully chosen vertices from a given near-Ramanujan graph and add a matching between their neighbors to maintain regularity. A crucial point in the spectral analysis here is the delocalization of the eigenvectors of the graphs obtained, which is based on the absence of short cycles in the neighborhoods of the omitted vertices. 
The rest of this paper is organized as follows. In Section 2 we describe the strongly explicit constructions, including the proofs of Proposition 1.1 and Theorem 1.2, In Section 3 we present the proof of Theorem [1.3. The final Section 4 contains some concluding remarks and open problems.

\section{Strongly explicit constructions}

The basic construction we describe here requires the ability to find efficiently a large prime in a prescribed range. It is well known that this can be done efficiently by a randomized algorithm, and can also be done deterministically assuming some standard (open) numbertheoretic conjectures about the gap between consecutive primes. Since this is the only non-deterministic part of the construction, we call it a $p$-strongly explicit construction (where $p$ stands for prime). This construction is described in the first subsection. We then show how it can be replaced by a totally strongly explicit construction. To do so, we first include a subsection presenting the (known) description of the construction of [16], [17] as Cayley graphs of Quaternions over $Z_{m}$. We proceed with a proof of Theorem 1.2 with a $p$-strongly explicit construction, followed by its modification to a strongly explicit one.

\subsection{The basic construction}

We start with the simple proof of Proposition 1.1, with a $p$-strongly explicit construction. It is based on the fact that if $G_{i}=\left(V, E_{i}\right), i \in I$, are graphs on the same set of vertices $V$, where $G_{i}$ is an $\left(n, d_{i}, \lambda_{i}\right)$-graph, then their union $G=\left(V, \cup_{i} E_{i}\right)$ (considered as a multigraph in case the sets $E_{i}$ are not pairwise disjoint), is an $\left(n, \sum_{i} d_{i}, \sum_{i} \lambda_{i}\right)$ graph. This is a simple consequence of the variational definition of the eigenvalues. The Ramanujan graphs in [16] or [17] are Cayley graphs of the group $S L\left(2, F_{q}\right)$ of the two by two matrices with determinant 1 over the finite field $F_{q}$, modulo its normal subgroup consisting of the identity $I$ and $-I$. The degree can be 1 plus any prime $p$ congruent to 1 modulo 4 , where $q$ is also a prime congruent to 1 modulo 4 , and $p$ is a quadratic residue modulo $q$. Note that by quadratic reciprocity this is equivalent to $q$ being a quadratic residue modulo $p$.

Given a desired degree $d=d_{1}$, let $p_{1}$ be the largest prime congruent to 1 modulo 4 and satisfying $p_{1}+1 \leq d_{1}$. Put $d_{2}=d_{1}-p_{1}-1$. If $d_{2}>4$ let $p_{2}$ be the largest prime congruent to 1 modulo 4 which satisfies $p_{2}+1 \leq d_{2}$ and put $d_{3}=d_{2}-p_{2}-1$. Continuing in this manner we get primes $p_{1}, \ldots, p_{s}$ as above so that $\left(p_{1}+1\right)+\left(p_{2}+1\right)+\cdots+\left(p_{s}+1\right) \leq d$ where $y=d-\left(\left(p_{1}+1\right)+\left(p_{2}+1\right)+\cdots+\left(p_{s}+1\right)\right) \leq 4$. Let $q$ be a prime congruent to 1 modulo 4 which is a quadratic residue modulo each $p_{i}$ (for example, any $q$ which is 
1 modulo each $p_{i}$ will do). Let $V$ be the set of elements of $S L\left(2, F_{q}\right)$. For each $i$ let $G_{i}$ be the $\left(p_{i}+1\right)$-regular Ramanujan Cayley graph of $S L\left(2, F_{q}\right)$ described in [16], and let $X_{i}$ be its (symmetric) set of generators. Let $G^{\prime}$ be the Cayley graph of $S L\left(2, F_{q}\right)$ whose set of generators consists of the union of all sets $X_{i}$. Then $G^{\prime}$ is $(d-y)$-regular, where $0 \leq y \leq 4$. If $y=0$ let $G$ be $G^{\prime}$. If $y=1$ add to the set of generators the matrix $M$ with rows $(0,1)$ and $(-1,0)$ (which is of order 2 ). If $y=2$ add an arbitrary generator and its inverse, if $y=3$ add such a generator, its inverse and $M$, and if $y=4$ add an arbitrary set of two generators and their inverses. In each of these cases the resulting graph $G$ is a $d$-regular Cayley graph of $S L\left(2, F_{q}\right)$. By the known results about the distribution of primes in arithmetic progressions each prime $p_{i}$ is much smaller than $p_{i-1}$ as long as $p_{i-1}$ is large. In fact, by [4] it follows that $p_{i}=O\left(p_{i-1}^{0.525}\right)$. Therefore, the resulting graph $G$ is an $(n, d, \lambda)$-graph for $n=q\left(q^{2}-1 / 2\right.$ with $\lambda \leq\left(2+o_{d}(1)\right) \sqrt{d}$, where the $o(1)$-term tends to zero as $d$ tends to infinity. Note that it is not difficult to ensure, if so desired, that the graph $G$ is simple: we just have to ensure the chosen primes are distinct. This is automatically the case whenever $d_{i}$ is still large, and if needed we can stop when $d_{i}$ becomes small and add arbitrary additional generators and their inverses, together with $M$ if $d$ is odd. Alternatively, if we have to repeat the same prime several times, we can take the corresponding generating set for this prime and conjugate it to get an isomorphic graph with different generators. The known results about the distribution of primes in arithmetic progressions imply also that for each choice of the primes $p_{i}$ the possible choices for the prime $q$ suffice to ensure that the sequence of possible values for the number of vertices $n$ of the graph is one in which the ratio between consecutive terms tends to 1 as $n$ tends to infinity. This completes the proof of the proposition (with a $p$-strongly explicit construction resulting from the need to find the required large prime $q$ ).

\subsection{Ramanujan graphs as Cayley graphs of quaternions}

In this subsection we present the known description of the LPS Ramanujan graphs as Cayley graphs of quaternions. The proof these are Ramanujan graphs appears (somewhat implicitly) in [15].

Let $p$ be a prime congruent to 1 modulo 4 , and let $A=A(p)$ be the set of all integral solutions $\left(a_{0}, a_{1}, a_{2}, a_{3}\right)$ of the equation $a_{0}^{2}+a_{1}^{2}+a_{2}^{2}+a_{3}^{2}=p$ where $a_{0}$ is positive odd, and all other $a_{i}$ are even. By a well known result of Jacobi there are exactly $p+1$ such vectors. Let $m$ be odd, relatively prime to $p$, and assume further that $p$ is a square in $Z_{m}^{*}$. let $Q(m)$ be the factor group of the multiplicative group of the quaternions over $Z_{m}$ whose norm 
is a square in $Z_{m}^{*}$, modulo its normal subgroup consisting of the scalars $Z_{m}^{*}$. Thus the elements of $Q(m)$ are all quaternions $x_{0}+x_{1} i+x_{2} j+x_{3} k$ where $x_{0}^{2}+x_{1}^{2}+x_{2}^{2}+x_{3}^{2} \in\left(Z_{m}^{*}\right)^{2}$ and two such elements are identified if one is a multiple of the other by a scalar. Finally, let $H=H(p, m)$ be the Cayley graph of $Q(m)$ with the generating set

$$
\left\{a_{0}+a_{1} i+a_{2} j+a_{3} k:\left(a_{0}, a_{1}, a_{2}, a_{3}\right) \in A(p)\right\} .
$$

The following result is proved (somewhat implicitly) in [15], see pages 95-97.

Theorem 2.1 ([15]). For every $p$ and $m$ as above $H=H(p, m)$ is a non-bipartite $(p+1)$ regular Ramanujan graph, that is, the absolute value of each of its eigenvalues besides the top one is at most $2 \sqrt{p}$.

\subsection{The proof of Proposition 1.1}

In the construction here we will start with the graphs $Q(p, m)$ with $p \equiv 1 \bmod 4$ a prime and $m=q_{1}^{s} q_{2}^{t}$, where $s, t \geq 1$ and $q_{1}, q_{2}$ are distinct primes, each being $1 \bmod 4 p$. For each fixed $p$ as above, the known results about the Linnik problem (see [11]) imply that there are $q_{1}, q_{2}$ as above, each being at most a polynomial in $p$. It is not difficult to check, using Hensel's Lemma and the Chinese Remainder Theorem, that the number of vertices of $H\left(p, q_{1}^{s} q_{2}^{t}\right)$ is

$$
Q\left(q_{1}, q_{2}, s, t\right)=q_{1}^{3(s-1)} q_{2}^{3(t-1)} \frac{q_{1}\left(q_{1}-1\right)\left(q_{1}+1\right)}{2} \frac{q_{2}\left(q_{2}-1\right)\left(q_{2}+1\right)}{2} .
$$

Indeed, by Hensel's Lemma, for elements $x_{0}, x_{1}, x_{2}, x_{3}$ of $Z_{m}$ the norm $x_{0}^{2}+x_{1}^{2}+x_{2}^{2}+x_{3}^{2}$ is a square in $Z_{m}^{*}$ if and only if it is a square in $Z_{q_{1}}^{*}$ and in $Z_{q_{2}}^{*}$. Since each $q_{i}$ is $1 \bmod 4$, -1 is a quadratic residue implying that the number of solutions of $y_{1}^{2}+y_{2}^{2}=0$ in $Z_{q_{i}}$ is $2 q_{i}-1$. For each nonzero $b$ in $Z_{q_{i}}$ the number of solutions of $y_{1}^{2}+y_{2}^{2}=b$ (in $Z_{q_{i}}$ ) is the same as the number of solutions of $y^{2}-z^{2}(=(y-z)(y+z))=b$, which is $q_{i}-1$. This shows that the number of solutions of $x_{0}^{2}+x_{1}^{2}+x_{2}^{2}+x_{3}^{2}=b$ for any nonzero $b \in Z_{q_{i}}$ is

$$
2\left(2 q_{i}-1\right)\left(q_{i}-1\right)+\left(q_{i}-2\right)\left(q_{i}-1\right)^{2}=\left(q_{i}-1\right) q_{i}\left(q_{i}+1\right) .
$$

(These include $\left(2 q_{i}-1\right)\left(q_{i}-1\right)$ solutions with $x_{0}^{2}+x_{1}^{2}=0$ and $x_{2}^{2}+x_{3}^{2}=b,\left(2 q_{i}-1\right)\left(q_{i}-1\right)$ ones with $x_{0}^{2}+x_{1}^{2}=b$ and $x_{2}^{2}+x_{3}^{2}=0$, and $\left(q_{i}-1\right)^{2}$ solutions for each of the $q_{i}-2$ possibilities $x_{0}^{2}+x_{1}^{2}=b_{1}$ and $x_{2}^{2}+x_{3}^{2}=b_{2}$ with $b_{1}+b_{2}=b$ and $b_{1}, b_{2} \notin\{0, b\}$.) Therefore, the number of elements over $Z_{q_{i}}$ whose norm is a nonzero square in $Z_{q_{i}}$ is

$$
\frac{q_{i}-1}{2}\left(q_{i}-1\right) q_{i}\left(q_{i}+1\right)=\frac{q_{i}\left(q_{i}-1\right)^{2}\left(q_{i}+1\right)}{2} .
$$


By the Chinese remainder Theorem there are

$$
\frac{q_{1}\left(q_{1}-1\right)^{2}\left(q_{1}+1\right)}{2} \frac{q_{2}\left(q_{2}-1\right)^{2}\left(q_{2}+1\right)}{2} .
$$

elements $\left(x_{0}, x_{1}, x_{2}, x_{3}\right)$ in $Z_{q_{1} q_{2}}$ so that $x_{0}^{2}+x_{1}^{2}+x_{2}^{2}+x_{3}^{2}$ is a square in $Z_{q_{1} q_{2}}^{*}$, and by Hensel's Lemma each of them provides $q_{1}^{4(s-1)} q_{2}^{4(t-1)}$ quaternions over $Z_{q_{1}^{s} q_{2}^{t}}$ whose norm is a square in $Z_{q_{1}^{s} q_{2}^{t}}^{*}$. To get the number of vertices of the graph we just have to divide by the cardinality of $Z_{q_{1}^{s} q_{2}^{t}}^{*}$ which is $q_{1}^{s-1} q_{2}^{t-1}\left(q_{1}-1\right)\left(q_{2}-1\right)$, obtaining the required number of vertices. Note also that by this description it is easy to number the vertices of the graph. (For our application here it is in fact enough to number a constant fraction of them. For fixed $q_{1}, q_{2}$ this can be done, for example, by numbering all vectors $\left(1, x_{1}, x_{2}, x_{3}\right) \in Z_{q_{1}^{s} q_{2}^{t}}$ with $x_{1}, x_{2}, x_{3}$ divisible by $q_{1} q_{2}$, lexicographically).

We next show that for every fixed distinct primes $q_{1}, q_{2}$, the ratio between consecutive elements in the set of integers $\left\{Q\left(q_{1}, q_{2}, s, t\right): s, t \geq 1\right\}$ tends to 1 as the elements grow.

Lemma 2.2. Let $q_{1}, q_{2}$ be distinct primes. Then for every large integer $n$ there are positive integers $s, t$ so that $n \leq Q\left(q_{1}, q_{2}, s, t\right) \leq n+o(n)$.

Proof: The constant $\alpha=\frac{\log q_{1}}{\log q_{2}}$ is irrational. Therefore, by the equidistribtion theorem (in fact, by a special case that follows easily from the pigeonhole principle), for every $\delta>0$ there is an integer $k_{1}=k_{1}(\alpha)$ so that $0<k_{1} \alpha \bmod 1<\delta$. It follows that for every $\mu>0$ there are integers $k_{1}, k_{2}$ such that

$$
1 \leq \frac{q_{1}^{k_{1}}}{q_{2}^{k_{2}}} \leq q_{2}^{\delta} \leq(1+\mu) .
$$

This implies that for every $s, t \geq \max \left\{k_{1}, k_{2}\right\}$ the ratio between $Q\left(q_{1}, q_{2}, s, t\right)$ and $Q\left(q_{1}, q_{2}, s-\right.$ $\left.k_{1}, t-k_{2}\right)$ is between 1 and $(1+\mu)^{3}$, implying the desired result.

The proof of Proposition 1.1 now proceeds exactly as in subsection 2.1, using the description of the LPS graphs serving as the building blocks as given in subsection 2.2. Since here $q_{1}, q_{2}$ are constants, there is no need to find any large primes for the construction, providing a strongly explicit construction for every fixed degree.

\subsection{The proof of Theorem 1.2}

We first describe a $p$-strongly-explicit construction, starting, again, with the graphs of [16]. Recall that the vertex sets of these graphs is the set of matrices in $S L\left(2, F_{q}\right)$ where each matrix $A$ is identified with $-A$. It is easy to number the vertices starting with the 
matrices $\left(a_{i j}\right)$ with $a_{11} \neq 0$ and ordering them according to the lexicographic order of the elements $\left(a_{11}, a_{12}, a_{13}\right)$ where $a_{14}$ is chosen to ensure that the determinant is 1 (which is always possible as $\left.a_{11} \neq 0\right)$. Here $1 \leq a_{11} \leq(q-1) / 2$, as we identify each matrix $A$ with $-A$. The first matrices are the $q^{2}$ matrices with $a_{11}=1$, then those with $a_{11}=2$, and so on. (The remaining $q(q-1) / 2$ matrices with $a_{11}=0$ can appear last in our order according to the lexicographic order of $\left(a_{12}, a_{24}\right)$, but this will play no real role in the construction.)

Given the desired number $n$ of vertices, and given the degree $d=p+2$ with $p$ as in the theorem, let $q$ be the largest prime which is 1 modulo 4 , is a quadratic residue modulo $p$ and satisfies $\left|S L\left(2, F_{q}\right)\right|=m_{q}=q\left(q^{2}-1\right) / 2 \leq n$. Put $m=m_{q}$, let $H$ be the Ramanujan $(p+1)=(d-1)$-regular graph of [16] whose vertex set $V$ is the set of elements of $S L\left(2, F_{q}\right)$ numbered as described above. By the known results about the distribution of primes in progressions $n-m=o(m)$. Put $r=n-m$ and let $R$ be a set of $r$ additional vertices $u_{1}, u_{2}, \ldots, u_{r}$. Connect each vertex $u_{i}$ to the vertices numbered $(i-1) d+1,(i-1) d+2, \ldots, i d$ of $H$. Finally add a loop to each remaining vertex of $H$ to make the graph regular. This is the desired graph $G$. It is clearly $d=p+2$-regular. (If $n$ is even and we do not want loops we can replace them by a matching between consecutive pairs of vertices, saturating all non-neighbors of the $r$ new vertices).

It is clear that the construction above is strongly explicit. To complete the proof it remains to show that the absolute value of any nontrivial eigenvalue of $G$ is at most $\sqrt{2(p+1)}+\sqrt{p}+o(1)$. We proceed with a proof of this fact. By the variational definition of the nontrivial eigenvalues of $G$ this is equivalent to showing that for every real function $f(u)$ on the set of vertices $U=V \cup R$ of $G$ satisfying $\|f\|_{2}^{2}=1$ and $\sum_{u \in U} f(u)=0$

$$
\left|f^{t} A_{G} f\right| \leq \sqrt{2(p+1)}+\sqrt{p}+o(1)
$$

where $A_{G}$ is the adjacency matrix of $G$. Let $W \subset V$ denote the set of all $(p+2) r$ neighbors of $R$, put $L=V-W$, and let $E_{R}$ denote the set of all edges between $R$ and $W$. Thus $E_{R}$ is a collection of pairwise vertex disjoint stars, each having $(p+2)$ leaves. The adjacency matrix of $G$ can be written as a sum $A_{G}=A_{H}+A_{R}+A_{L}$, where $A_{H}$ is the adjacency matrix of the Ramanujan graph $H$ (with the additional isolated vertices of $R$ ), $A_{R}$ is the adjacency matrix of the graph $\left(U, E_{R}\right)$, and $A_{L}$ is the adjacency matrix of the graph on $U$ whose edges are the loops on the vertices of $L$ (or the added matching on them, if we have chosen not to add loops). Therefore

$$
f^{t} A_{G} f=f^{t} A_{H} f+f^{t} A_{R} f+f^{t} A_{L} f .
$$

We proceed to bound each of these terms. 
By Cauchy-Schwarz

$$
\left|\sum_{u \in R} f(u)\right|^{2} \leq|R| \sum_{u \in R} f^{2}(u) \leq|R|=o(m)
$$

Since $\sum_{u \in U} f(u)=0$, this implies that $\left|\sum_{u \in V} f(u)\right|^{2}=\left|\sum_{u \in R} f(u)\right|^{2}=o(m)$

Let $g$ be the trivial normalized eigenvector of $H$, that is, the vector given by $g(v)=$ $1 / \sqrt{m}$ for all $v \in V$. Expressing the restriction $f^{\prime}$ of $f$ to $V$ as a linear combination of $g$ and a unit vector $h$ orthogonal to it, we get $f^{\prime}=b g+c h$, where $\sum_{v \in V} h(v)=0, b^{2}+c^{2}=1$ and $b^{2}=\left|\sum_{u \in V} f(u)\right|^{2} / m=o(1)$. Since $H$ is a Ramanujan graph, $\left|h^{t} A_{H} h\right| \leq 2 \sqrt{p}$. Therefore

$$
\left|f^{t} A_{H} f\right|=\left|\left(f^{\prime}\right)^{t} A_{H} f^{\prime}\right| \leq b^{2}(p+1)+c^{2} 2 \sqrt{p} \leq 2 \sqrt{p}+o(1) .
$$

Clearly

$$
\left|f^{t} A_{L} f\right| \leq \sum_{v \in L} f^{2}(v)
$$

Indeed this is an equality if there are loops and an inequality in case a matching has been added.

For bounding the absolute value of $f^{t} A_{R} f$ observe that for every positive $x$

$$
\begin{gathered}
\left|f^{t} A_{R} f\right|=2\left|\sum_{u v \in E_{R}} f(u) f(v)\right| \\
\leq \sum_{u \in R, v \in W, u v \in E_{R}}\left(\frac{f^{2}(u)}{x}+x f^{2}(v)\right)=\frac{p+2}{x} \sum_{u \in R} f^{2}(u)+x \sum_{v \in w} f^{2}(v) .
\end{gathered}
$$

Combining (2),(3), (44) and (5) we conclude that for every positive real $x$

$$
\left|f^{t} A_{G} f\right| \leq(2 \sqrt{p}+1) \sum_{v \in L} f^{2}(v)+(2 \sqrt{p}+x) \sum_{v \in W} f^{2}(v)+\frac{p+2}{x} \sum_{v \in R} f^{2}(v)+o(1) .
$$

Choosing $x=\sqrt{2 p+2}-\sqrt{p}$ (which is at least 1) and substituting in (마) we finally get

$$
\left|f^{t} A_{G} f\right| \leq(\sqrt{2 p+2}+\sqrt{p}) \sum_{u \in U} f^{2}(u)+o(1)=(\sqrt{2 p+2}+\sqrt{p})+o(1) .
$$

This establishes (1) and completes the proof (with a $p$-strongly explicit construction). The conversion to a strongly explicit construction proceeds just as in the proof of Proposition 1.1. based on the results in subsection 2.2. Note that as mentioned in that subsection the description there provides a simple efficient way to number enough vertices of each graph $H\left(p, q_{1}^{s} q_{2}^{t}\right)$ and by Lemma 2.2 we can start by finding efficiently appropriate $s, t$ using binary search. 


\section{Explicit constructions}

In this section we present the proof of Theorem 1.3. We start with some preliminary lemmas.

Lemma 3.1. Let $G=(V, E)$ be a d-regular graph on $n$ vertices, where $d \geq 3$, and suppose that the $2 r+4$-neighborhood of any vertex in it contains at most one cycle, where $r \leq \log _{d-1} n$. Then there is a subset $U \subset V$ of vertices of $G$ satisfying the following.

1. $|U| \geq \frac{n}{2 d^{2 r+3}}$

2. The $(r+1)$-neighborhood of any vertex in $U$ contains no cycle.

3. The distance between any two vertices in $U$ is at least $2 r+3$.

Such a set $U$ can be found in polynomial time.

Proof: Let $\mathcal{C}$ denote the collection of all cycles of length at most $2 r+4$ in $G$. Note that the distance between any two members $C_{1}, C_{2}$ of $\mathcal{C}$ is larger than $2 r+4$, since otherwise there is a vertex $v$ within distance at most $r+2$ of both cycles $C_{i}$, and then its $2 r+4$ neighborhood contains both cycles, contradiction. The $r+2$ neighborhood of each cycle $C \in \mathcal{C}$ contains no other cycle besides $C$, as it is contained in the $2 r+4$ neighborhood of any vertex on the cycle. Thus the number of edges spanned by each such neighborhood is at most the number of vertices in it. As the neighborhoods are vertex disjoint, the total number of edges in all these neighborhoods together is at most the number of vertices of $G$ which is $n$. It follows that by omitting all vertices in the $(r+1)$-neighborhoods of all members of $\mathcal{C}$, at most $n$ edges are omitted, and as $G$ has $n d / 2$ edges and $d \geq 3$ at least $n / 2$ edges, and hence at least $n / 2 d$ vertices have not been omitted. Let $Z$ be the set of non-omitted vertices. Note that the $(r+1)$-neighborhood of any vertex in $Z$ contains no cycle (as if it contains a cycle, it contains a cycle of length at most $2 r+3<2 r+4$ but the vertex is not within distance $r+1$ of any such cycle.) Starting with $U=\emptyset$ let $v_{1}$ be an arbitrary vertex of $Z$, add it to $U$ and remove all vertices of $Z$ within distance $2 r+2$ of $v_{1}$. Clearly at most $d^{2 r+2}$ vertices have been deleted. Let $v_{2}$ be an arbitrary vertex left in $Z$, add it to $U$ and remove all vertices of $U$ within distance $2 r+2$ of $v_{2}$. Continuing in this manner we get a set $U$ of at least $\frac{n}{2 d^{2 r+3}}$ vertices. It is clear that this set satisfies all the conclusions of the lemma. It is also clear that $U$ can be computed in polynomial time. 
The next lemma about the delocalization of eigenvectors of regular graphs in cycle-free neighborhood can be proved using the method of Kahale in [13] (see also [3] for a recent application of this technique). Here we present a simple self contained proof.

Lemma 3.2. Let $G=(V, E)$ be a d-regular graph where $d \geq 3$, let uv be an edge of $G$ and suppose that the $r$-neighborhood of uv contains no cycle. For each $i$ satisfying $0 \leq i \leq r$ let $N_{i}$ denote the set of all vertices of distance exactly $i$ from $\{u, v\}$. (In particular, $\left.N_{0}=\{u, v\}\right)$. Let $f$ be a nonzero eigenvector of $G$ with eigenvalue $\mu \geq 2 \sqrt{d-1}$. Then for every $1 \leq i \leq r$

$$
\sum_{w \in N_{i}} f^{2}(w) \geq \sum_{w \in N_{i-1}} f^{2}(w)
$$

Proof: We apply induction on $i$. Note that by assumption the induced subgraph of $G$ on the $r$-neighborhood of $u v$ is a $d$-regular tree. Therefore $\left|N_{i}\right|=2(d-1)^{i}$ for all $i \leq r$. Let $u_{1}, u_{2}, \ldots, u_{d-1}$ denote the neighbors of $u$ besides $v$, and let $v_{1}, v_{2}, \ldots, v_{d-1}$ denote the neighbors of $v$ besides $u$. Then

$$
f(v)+\sum_{i=1}^{d-1} f\left(u_{i}\right)=\mu f(u)
$$

and

$$
f(u)+\sum_{i=1}^{d-1} f\left(v_{i}\right)=\mu f(v)
$$

By Cauchy-Schwarz,

$$
f^{2}(v)+\sum_{i=1}^{d-1} f^{2}\left(u_{i}\right) \geq \frac{\mu^{2} f^{2}(u)}{d} \geq \frac{4 d-4}{d} f^{2}(u)
$$

and similarly

$$
f^{2}(u)+\sum_{i=1}^{d-1} f^{2}\left(v_{i}\right) \geq \frac{4 d-4}{d} f^{2}(v) .
$$

Summing, we conclude that

$$
f^{2}(u)+f^{2}(v)+\sum_{w \in N_{1}} f^{2}(w) \geq \frac{4 d-4}{d}\left(f^{2}(u)+f^{2}(v)\right)
$$

implying that

$$
\sum_{w \in N_{1}} f^{2}(w) \geq \frac{3 d-4}{d}\left(f^{2}(u)+f^{2}(v)\right) \geq f^{2}(u)+f^{2}(v)=\sum_{w \in N_{0}} f^{2}(w) .
$$


This proves (7) for $i=1$.

Assuming it holds for $i-1$ we prove it for $i$. For each vertex $w \in N_{i-1}$ let $w^{\prime}$ be its unique parent in $N_{i-2}$ and let $x_{1}, x_{2}, \ldots, x_{d-1}$ be its neighbors in $N_{i}$. Then

$$
f\left(w^{\prime}\right)+\sum_{i=1}^{d-1} f\left(x_{i}\right)=\mu f(w) .
$$

Since $f\left(w^{\prime}\right)=(d-1) \cdot \frac{f\left(w^{\prime}\right)}{d-1}$, we get, by Cauchy-Schwarz,

$$
\frac{f^{2}\left(w^{\prime}\right)}{d-1}+\sum_{i=1}^{d-1} f^{2}\left(x_{i}\right) \geq \frac{\mu^{2} f^{2}(w)}{2 d-2} \geq 2 f^{2}(w) .
$$

Summing the above inequality for all $w$ in $N_{i-1}$, each vertex $w^{\prime}$ appears in the LHS exactly $d-1$ times, yielding

$$
\sum_{w^{\prime} \in N_{i-2}} f^{2}\left(w^{\prime}\right)+\sum_{x \in N_{i}} f^{2}(x) \geq 2 \sum_{w \in N_{i-1}} f^{2}(w) .
$$

This gives

$$
\sum_{x \in N_{i}} f^{2}(x) \geq 2 \sum_{w \in N_{i-1}} f^{2}(w)-\sum_{w^{\prime} \in N_{i-2}} f^{2}\left(w^{\prime}\right) \geq \sum_{w \in N_{i-1}} f^{2}(w)
$$

where the last inequality follows from the induction hypothesis. This completes the proof of the induction step, establishing the assertion of the lemma.

Finally, we need the main result of Mohanty, O'Donnell and Paredes in [18], which is the following.

Theorem $3.3([18])$. For every $d, \epsilon>0$ and (large) $n$ there is an explicit construction of an $(n+o(n), d, \lambda)$-graph with $\lambda \leq 2 \sqrt{d-1}+\epsilon$ so that the $s$ neighborhood of any vertex contains at most one cycle, where $s \geq(\log \log n)^{2}$.

We note that the result is stated in [18] without the conclusion about the cycles, but the version above follows from the proof as presented there.

We are now ready to prove Theorem 1.3 .

Proof of Theorem 1.3: Put $r=\lceil 2 / \epsilon\rceil$ and $s=2 r+4$. Let $H=(V, E)$ be an explicit $(n+u, d, \lambda)$-graph with $u=o(n)$ and $\lambda \leq 2 \sqrt{d-1}+\epsilon / 2$ in which the $s$-neighborhood of any vertex contains at most one cycle. Such an $H$ exists by Theorem 3.3. By Lemma 3.1 we can find efficiently a set $U$ of $u$ vertices in $H$ satisfying the assertion of the lemma. Omit these vertices from the graph to get a graph $H^{\prime}$ and add a matching $M$ between 
their neighbors retaining the degree of regularity $d$. Let $G$ denote the resulting graph. Clearly it is $d$-regular and has $n$ vertices. Note that the $r$-neighborhood of any edge $u v$ of the added matching $M$ contains no cycle. In order to complete the proof it remains to show that every nontrivial eigenvalue of $G$ has absolute value at most $2 \sqrt{d-1}+\epsilon$. Let $A_{G}$ be the adjacency matrix of $G, A_{H^{\prime}}$ the adjacency matrix of $H^{\prime}$ (on the set of vertices $V)$ and $A_{M}$ the adjacency matrix of the graph on the set of vertices $V$ whose edges are those of the matching $M$. Note that $A_{G}=A_{H^{\prime}}+A_{M}$. Let $\lambda$ be a nontrivial eigenvalue of $G$ and let $f$ be a corresponding eigenvector satisfying $\sum_{v \in V} f^{2}(v)=1$. Then

$$
\lambda=f^{t} A_{G} f=f^{t} A_{H^{\prime}} f+f^{t} A_{M} f .
$$

Since $H^{\prime}$ is an induced subgraph of $H$ and all nontrivial eigenvalues of $H$ have absolute value at most $2 \sqrt{d-1}+\epsilon / 2$ it follows, by eigenvalue interlacing, that

$$
\left|f^{t} A_{H^{\prime}} f\right| \leq 2 \sqrt{d-1}+\epsilon / 2
$$

It is also clear that

$$
\left|f^{t} A_{M} f\right|=\left|2 \sum_{u v \in M} f(u) f(v)\right| \leq \sum_{u v \in M} f^{2}(u)+f^{2}(v) .
$$

If $|\lambda| \leq 2 \sqrt{d-1}$ there is nothing to prove, we thus assume that $\lambda \geq 2 \sqrt{d-1}$. Since the $r$-neighborhood of any edge of $M$ contains no cycle, Lemma 2.2 implies that for every such edge $u v$,

$$
f^{2}(u)+f^{2}(v) \leq \frac{1}{r} \sum_{w \in N(u, v, r)} f^{2}(w),
$$

where $N(u, v, r)$ denotes the $r$-neighborhood of $u v$. Since all these neighborhoods are pairwise disjoint it follows that

$$
\sum_{u v \in M} f^{2}(u)+f^{2}(v) \leq \frac{1}{r} \sum_{w \in V} f^{2}(v) \leq \frac{\epsilon}{2} .
$$

The desired result follows by plugging (9) and (11) in (8) (using (10)).

\section{Concluding remarks}

- Morgenstern [19] gave a strongly explicit construction of Ramanujan graphs for every degree which is a prime power plus 1 , but we cannot apply his construction in the proof of Proposition 1.1 since his construction provides Cayley graphs of different 
groups (and different sizes) for different degrees and hence one cannot pack the graphs corresponding to several degrees. Similarly, we cannot use his construction in the proof of Theorem 1.2 since for every fixed degree the sequence of possible numbers of vertices in his construction for this degree is too sparse.

- The proof of Theorem 1.3 can be applied directly to high girth Ramanujan graphs like those in [16], [17] in case the required degree is $p+1$ for a prime $p$ congruent to 1 mod 4 to obtain near Ramanujan graphs of this degree with any required (large) number of vertices.

- The problem of obtaining strongly explicit (two-sided) Ramanujan (and not nearly Ramanujan) graphs for any degree and number of vertices remains open.

Acknowledgment I thank László Babai, Oded Goldreich, Ryan O’Donnell, Ori Parzanchevski and Peter Sarnak for helpful discussions.

\section{References}

[1] N. Alon, Eigenvalues and expanders, Combinatorica 6(1986), 83-96.

[2] N. Alon and F. R. K. Chung, Explicit construction of linear sized tolerant networks, Discrete Math. 72 (1988), 15-19; (Proc. of the First Japan Conference on Graph Theory and Applications, Hakone, Japan, 1986.)

[3] N. Alon, S. Ganguly and N. Srivastava, High-girth near-Ramanujan graphs with localized eigenvectors, arXiv:1908.03694, 2019.

[4] R. C. Baker, G. Harman and J. Pintz, The difference between consecutive primes II., Proc. London Math. Soc., 83 (2001), no.3, 532-562.

[5] A. Ben-Aroya and A. Ta-Shma, A combinatorial construction of almost Ramanujan graphs using the zig-zag product, SIAM Journal on Computing 40(2), 267-290, 2011.

[6] C. Bordenave, A new proof of Friedman's second eigenvalue theorem and its extension to random lifts, arXiv 1502.04482v4, 2019. To appear in Annales scientifiques de lÉcole normale supérieure.

[7] S. M. Cioaba and M. Ram Murty, Expander graphs and gaps between primes, Forum Mathematicum 20(4), 745-756, 2008. 
[8] M. Cohen, Ramanujan graphs in polynomial time, In Proceedings of the 57th Annual IEEE Symposium on Foundations of Computer Science, pages 276-281, 2016.

[9] J. Friedman, Some geometric aspects of graphs and their eigenfunctions, Duke Mathematical Journal 69(3), 487-525, 1993.

[10] J. Friedman, A proof of Alon's second eigenvalue conjecture and related problems, Memoirs of the American Mathematical Society, 195(910), viii+100, 2008

[11] D. R. Heath-Brown, Zero-free regions for Dirichlet L-functions, and the least prime in an arithmetic progression, Proc. London Math. Soc. (3) 64, no. 2 (1992), 265-338.

[12] S. Hoory, N. Linial and A. Wigderson, Expander graphs and their applications, Bull. Amer. Math. Soc. (N.S.) 43 (2006), no. 4, 439-561.

[13] N. Kahale, Eigenvalues and expansion of regular graphs, Journal of the ACM 42(5), 1091-1106, 1995.

[14] M. Krivelevich and B. Sudakov, Pseudo-random graphs, in: More sets, graphs and numbers, 199-262, Bolyai Soc. Math. Stud., 15, Springer, Berlin, 2006.

[15] A. Lubotzky, Discrete Groups, Expanding Graphs and Invariant Measures, Springer 2010

[16] A. Lubotzky, R. Phillips and P. Sarnak, Ramanujan graphs, Combinatorica 8(3), 261-277, 1988.

[17] G. A. Margulis, Explicit group-theoretical constructions of combinatorial schemes and their application to the design of expanders and superconcentrators, Problemy Peredachi Informatsii 24(1988), 51-60 (in Russian). English translation in Problems of Information Transmission 24(1988), 39-46.

[18] S. Mohanty, R. O'Donnell and P. Paredes, Explicit near-Ramanujan graphs of every degree, arXiv 1909.06988v2

[19] M. Morgenstern, Existence and explicit constructions of $q+1$ regular Ramanujan graphs for every prime power $q$, Journal of Combinatorial Theory. Series B, 62(1), 44-62, 1994.

[20] A. Marcus, D. Spielman and N. Srivastava, Interlacing families I: Bipartite Ramanujan graphs of all degrees, Annals of Mathematics, Second Series, 182(1), 307-325, 2015 . 
[21] J. Murtagh, O. Reingold, A. Sidford and S. Vadhan, Deterministic Approximation of Random Walks in Small Space, Proc. APPROX-RANDOM 2019: 42:1-42:22.

[22] A. Nilli, On the second eigenvalue of a graph, Discrete Mathematics 91(2), 207-210, 1991.

[23] O. Reingold, S. Vadhan and A. Wigderson, Entropy waves, the zig-zag graph product, and new constant-degree expanders, Annals of Mathematics, 155(1), 157-187, 2002 